\title{
De nouveaux développements pour le trailtement du cancer par laser
}

\author{
Serge MORDON \\ INSERM U 703 - 59120 LOOS \\ serge.mordon@inserm.fr - www.u703.fr
}

Plusieurs programmes de recherche concernant le traitement du cancer par laser sont actuellement en cours au sein de l'unité INSERM U703 (CHRU de Lille), dédiée aux thérapies interventionnelles assistées par l'image et la simulation. Des techniques innovantes d'illumination intracavitaire sont développées pour le traitement par thérapie photodynamique des carcinoses péritonéales et du mésothéliome pleural malin. L'amélioration de la prise en charge des gliomes cérébraux de hauts grades au travers de nouveaux outils d'analyse d'image et de guidage va permettre de proposer deux nouvelles modalités de traitement reposant sur la thérapie photodynamique : interstitielle (iPDT) via l'insertion de fibre optique en condition stéréotaxique et peropératoire (perPDT) lors de la résection fluoroguidée. Enfin, l'étude et le développement de techniques de détection, de caractérisation et de quantification précises du cancer de la prostate devraient ouvrir la voie à des thérapies focalisées innovantes notamment par thermothérapie interstitielle par laser (LITT) et par photothérapie dynamique (PDT).

\section{Thérapie photodynamique du mésothéliome pleural malin}

\section{Contexte et enjeux du projet MesoPDT}

Considéré auparavant comme un cancer rare, le mésothéliome pleural malin (MPM) ou cancer primitif de la plèvre, est devenu un enjeu important devant son pronostic sombre (médiane de survie inférieure à un an dans la littérature) et son incidence croissante depuis 1945 entretenue par le large recours à l'amiante jusqu'à son interdiction en 1997. En France, l'incidence annuelle actuelle du mésothélium est évaluée à environ 900 cas (figure 1). Compte tenu du délai de latence long habituel entre l'exposition à l'amiante et le diagnostic du mésothéliome (en moyenne 30 à 40 ans), un pic d'incidence du MPM est attendu dans la prochaine décennie.

L'intérêt médical pour ce cancer a également évolué, passant d'un fatalisme quasiment exclusif à un réel investissement dans la prise en charge de cette maladie avec la notion de chirurgie acceptable, de nouvelles drogues cytotoxiques et d'associations efficaces, de l'émergence des biothérapies, de nouvelles modalités de radio- thérapie et surtout du développement de stratégies multidisciplinaires concertées qui permettent actuellement de construire des essais prospectifs, comparatifs, randomisés qui faisaient largement défaut dans ce domaine. Contribuant probablement au mauvais pronostic des patients et limi- tant souvent les options thérapeutiques, le MPM se caractérise aussi par des difficultés importantes dans son diagnostic.

En janvier 2012, a été décidée la constitution d'un réseau clinique français de centres experts (inter)régionaux pour la prise en charge du MPM (appelé « MESOCLIN »).

Estimation annuelle du nombre de cas incidents et du taux d'incidence du mésothéliome en France, chez les hommes et les femmes (pour 100 000) - Années 1998 - 2006.

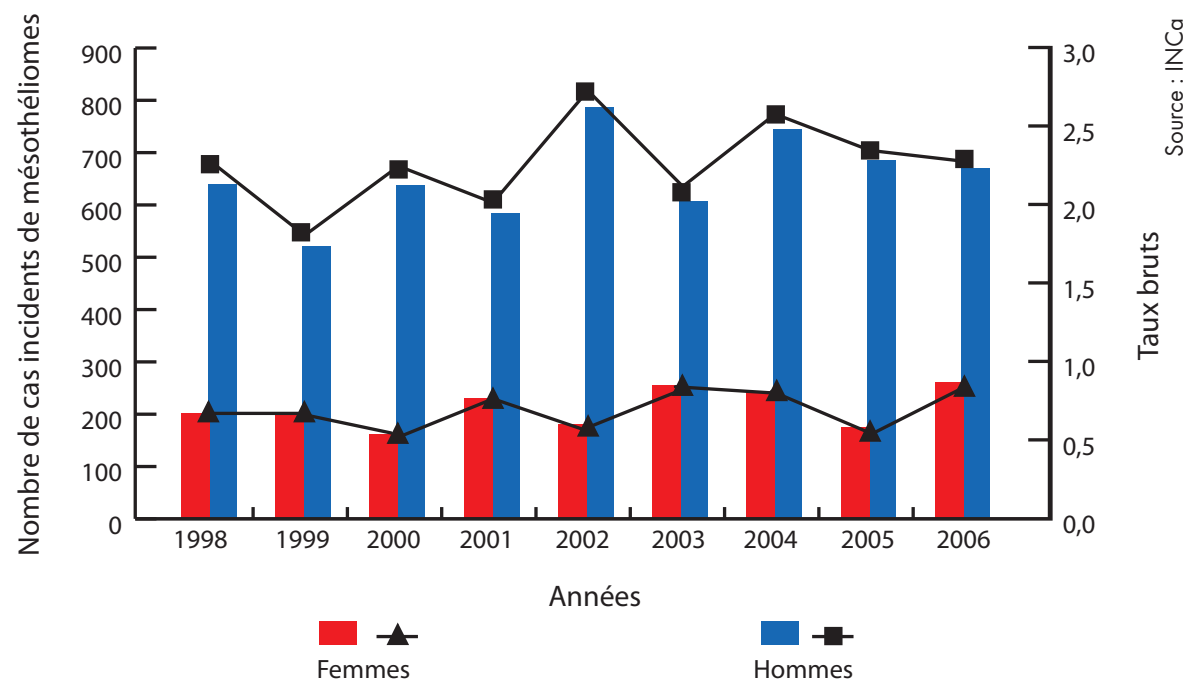

Figure 1. Amiante et mésothéliome pleural malin : état des connaissances en date du 19 janvier 2012. 
Ce réseau est financé par l'Institut national contre le cancer (INCa) et coordonné par le centre expert national basé à Lille, en étroite collaboration avec le réseau Mesopath (registre national ana-path des mésothéliomes).

\section{Utilisation de la thérapie photodynamique : des résultats prometteurs}

La thérapie photodynamique, ou PDT, est un traitement relativement nouveau dans le traitement du MPM, basé sur l'hypothèse que les cellules tumorales, si elles sont d'abord traitées par un agent photosensibilisant, mourront quand elles seront exposées à une lumière émise par une source laser à une longueur d'onde spécifique. Après une résection tumorale la plus complète possible, le chirurgien illumine avec la lumière (laser) directement la cavité pleurale produisant une lumière spécifiquement absorbée par la substance photosensibilisante. Quand les cellules tumorales absorbent la lumière, la drogue photosensibilisante produit des radicaux libres de l'oxygène qui tuent les cellules tumorales environnantes et l'angiogenèse tumorale.

Ainsi la PDT a-t-elle été testée dans des essais cliniques de phase I et II pour les patients MPM en combinaison avec la PEP (pneumonectomie extrapleurale) ou la PR (pleurectomie), et une chimiothérapie. Si les données préliminaires ont été assez décevantes, une étude récente par Friedberg [1] a obtenu des résultats très prometteurs par la combinaison d'une PR (mais pas de la PEP), d'une PDT intra-opératoire et d'une chimiothérapie adjuvante (cisplatine pemetrexed), avec une survie globale médiane de 31 mois dans une série incluant même des patients avec une forme localement avancée de MPM (figure 2). L'effet indésirable majeur potentiel de la PDT, la photosensibilisation, n'a pas entraîné de complications significatives dans cette série.

\section{L'illumination avec des textiles lumineux : un projet innovant}

Les cavités pleurales sont très complexes à illuminer. La technique actuelle consiste à remplir la cavité d'un liquide diffusant puis à procéder à l'illumination en « remuant » la source de lumière. Le contrôle de la dose de lumière pose un problème majeur et la difficulté d'assurer une bonne reproductibilité de la dose de lumière est responsable de récidives du MPM.

De récents travaux de l'équipe INSERM U 703 sur l'animal sur un modèle de carcinose péritonéale ont pu montrer que des illuminateurs souples permettaient d'assurer une illumination homogène (figure 3) [2,3]. Une collaboration avec I'ENSAIT (École nationale supérieure des arts et industries textiles) a permis de concevoir des textiles lumineux de grandes dimensions, parfaitement adaptés à l'illumination de la cavité pleurale. Le transfert industriel est en cours [4].

\section{Thérapies photodynamiques des gliomes de haut grade}

\section{Contexte et enjeux du projet NeuroPDT}

Avec une incidence de quatre nouveaux cas par an pour 100000 habitants soit 2400 nouveaux cas par an en France, le glioblastome, tumeur maligne du cerveau de grade élevé, est la troisième cause de décès par cancer chez l'adulte. II s'agit d'une tumeur incurable dont la médiane de survie est inférieure à un an. Même dans les situations les plus favorables, la plupart des patients décèdent dans les deux ans.

Ce domaine de l'oncologie est l'objet de nombreux travaux de recherches fondamentale et clinique. Le traitement de référence s'appuie sur l'exérèse chirurgicale suivie d'une radiothérapie cérébrale associée à une chimiothérapie concomitante et adjuvante par témozolomide. Néanmoins, le caractère invasif et rapidement proliférant du glioblastome ne permet pas son contrôle par les protocoles thérapeutiques conventionnels. De fait, les patients dont la localisation tumorale

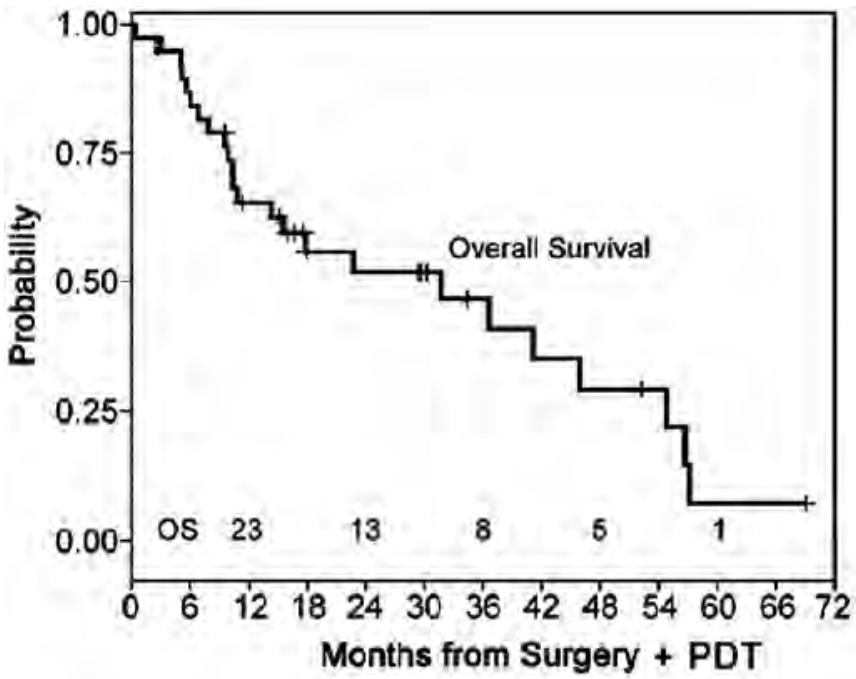

Figure 2. Courbe de survie des patients atteints de MPM et traités par PR + PDT intra-opératoire + chimiothérapie [1].

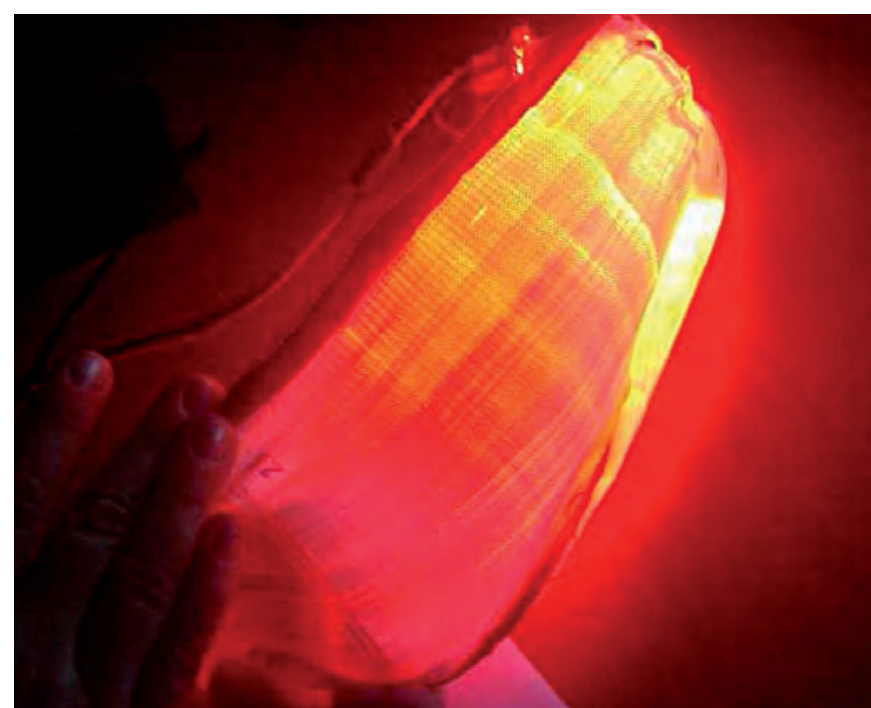

Figure 3. Illuminateur souple permettant une illumination homogène de la cavité pleurale. 
ne permet pas une exérèse chirurgicale présentent une survie limitée.

L'objectif de la recherche est de développer un schéma de thérapie photodynamique efficace. Si le travail expérimental confirme l'efficacité et l'innocuité de cette thérapie, celle-ci pourrait faire l'objet d'applications en clinique humaine.

En effet, la PDT, administrée soit par des fibres laser implantées en conditions stéréotaxiques, soit par illumination de la cavité opératoire, pourrait représenter une option thérapeutique pour les patients présentant un glioblastome inopérable ou en récidive. L'évaluation de ce développement clinique pourrait faire l'objet d'un programme de recherche clinique [5].

\section{Vers deux modalités distinctes de traitement}

La finalité du projet est de proposer deux modalités distinctes de traitement: la thérapie photodynamique per-opératoire (perPDT) et la thérapie photodynamique interstitielle (iPDT). En effet, ces deux solutions sont complémentaires puisque la perPDT est un complément thérapeutique prometteur pour enrichir l'arsenal thérapeutique actuel (exérèseradiothérapie-chimiothérapie) et l'iPDT constitue une modalité nouvelle pour la prise en charge des glioblastomes nonopérables.

L'étude pré-clinique est réalisée sur un modèle animal. La technique consiste en l'induction de tumeurs intracérébrales chez le rat en conditions stéréotaxiques. Les travaux menés au CRAN à Nancy, ont d'ores et déjà validé la faisabilité du concept qui consiste à réaliser l'iPDT guidée par IRM en stéréotaxie (figure 4). Ces fibres optiques couplées à une diode laser permettent une illumination focalisée de la zone tumorale. Réalisées au département hospitalo-universitaire de recherche expérimentale de Lille (DHURE), I'enjeu de ces expérimentations est multiple. Tout d'abord, cette étude doit permettre d'évaluer le bénéfice en termes de survie du traitement du glioblastome chez le rat. Sur
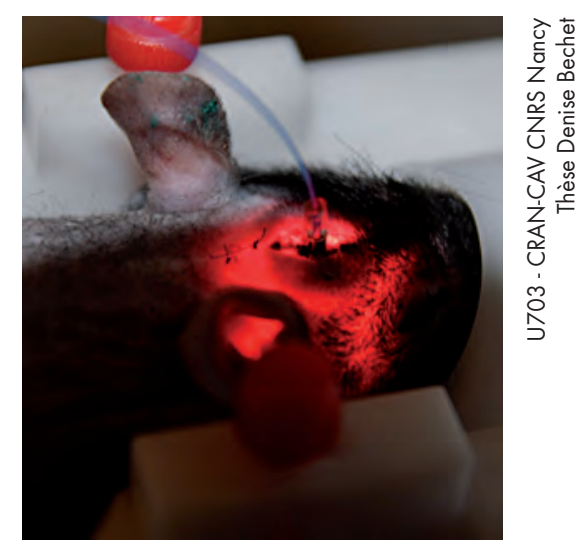

Figure 4. Fibre laser diffusante implantée en conditions stéréotaxiques.

un autre plan, cette étude doit permettre d'optimiser les modalités de traitement, de maîtriser l'ensemble des éléments de la chaîne de traitement (planification - traitement - suivi) et finalement de valider la preuve de concept de la PDT dans ce cadre.

L'étude clinique doit valider d'une part le concept de fixation sélective de l'agent

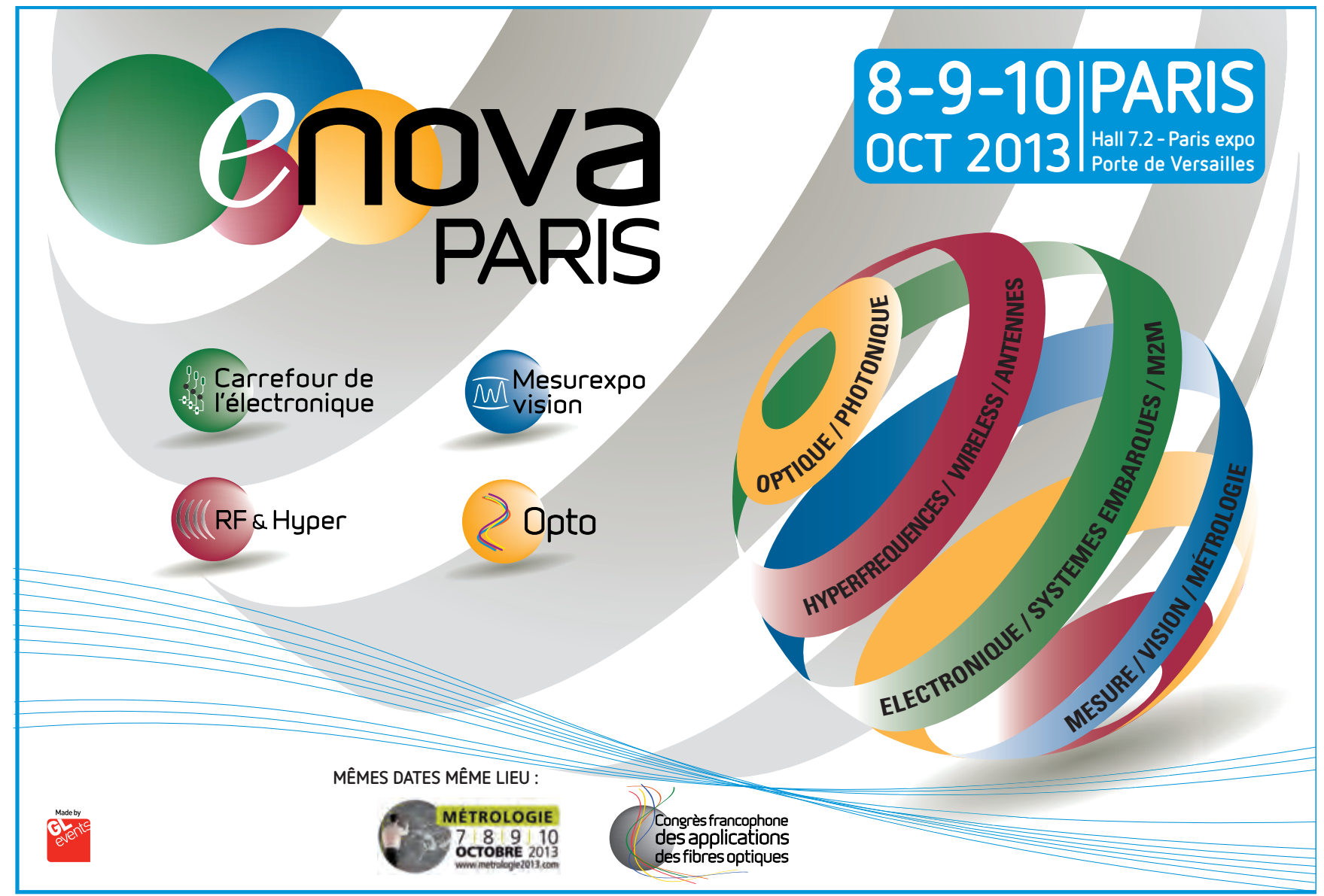


pharmacologique et, d'autre part, la mise en place d'un protocole d'imagerie associée à la prise en charge par PDT. La première phase consiste alors à mettre en place localement un protocole de résection fluoroguidée. À partir de pièces d'exérèse lors de ces résections guidées par fluorescence, l'étude d'anatomopathologie permettra de vérifier la sélectivité de ce produit et sa concentration en fonction des différents grades observés. Cette étude est cruciale car le passage à la clinique ne sera réalisable que sur la base des résultats obtenus dans cette phase préliminaire. En effet, la sélectivité doit garantir l'innocuité du produit en dehors de la cible et ainsi éviter toute exposition de tissus sains. À plus long terme, une étude approfondie des techniques d'imagerie sera mise en place afin de définir les meilleures solutions pour la planification, le guidage et le suivi du traitement.

Les développements méthodologiques concernent à la fois le traitement de l'image et les modalités de traitement en iPDT ou perPDT, tels que des illuminateurs spécifiques (figure 5).

\section{Ciblage focal du cancer de la prostate par laser interstitiel}

\section{Contexte et enjeux du projet PCaFLA}

Le cancer de la prostate (PCa) est le premier cancer chez l'homme de plus de 50 ans dans les pays industrialisés. L'incidence croissante de ce cancer $(+8,5 \%$ entre 2000 et 2010 en France) est rapportée au vieillissement de la population et à la diffusion du dépistage individuel.

Pour l'association française d'urologie, les recommandations thérapeutiques actuelles du cancer de la prostate localisé, consistent en une approche radicale visant à traiter toute la glande. Le développement de nouvelles alternatives thérapeutiques permettant un traitement focal des lésions cancéreuses au sein de la prostate est aujourd'hui discuté.

Différentes formes d'énergie sont en développement pour réaliser ces thérapies focales. Le laser interstitiel pourrait être une solution adaptée à un organe

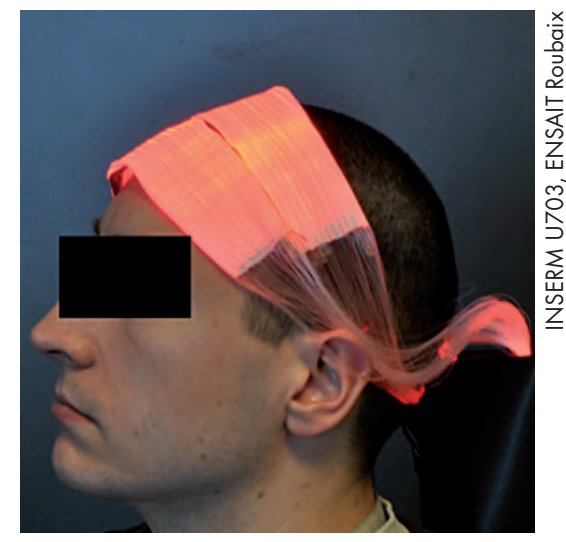

Figure 5. Illuminateur spécifique pour la perPDT : fibres optiques tissées pilotées par microcontrôleur.

confiné comme la prostate. Les thérapies laser peuvent se faire de deux façons :

- la thérapie photodynamique (PDT) comme décrit précédemment ;

- la thérapie thermique (LITT) qui consiste en l'induction d'une nécrose tissulaire par thermothérapie à l'aide de fibres laser diffusantes placées dans la prostate.

Cependant, l'essor de telles techniques ne peut se réaliser sans le développement d'outils pour le diagnostic et la cartographie précise des zones cancéreuses dans la glande. L'IRM multiparamétrique (T1W, T2W, perfusion et diffusion) constitue un moyen aujourd'hui reconnu pour la détection de lésions naissantes dans la prostate [6].

Les objectifs des recherches en cours sont de développer des outils d'aide au diagnostic par ordinateur (computer aided diagnosis) du cancer de la prostate en utilisant l'IRM multiparamétrique et la mise en place de plateformes de thérapie par laser interstitiel efficace et reproductible. Les travaux concernent également la définition des critères d'éligibilité et d'inclusion qui permettront de proposer ce type de traitements aux cas les plus adaptés.

\section{Des réalisations et un projet...}

De par ses activités précédentes, I'unité INSERM U 703 bénéficie d'une forte expérience dans le domaine du traitement d'images pour la planification et le guidage de la radiothérapie conformationnelle du cancer de la prostate. Cet acquis a été exploité pour le développement d'outils informatiques pour le traitement

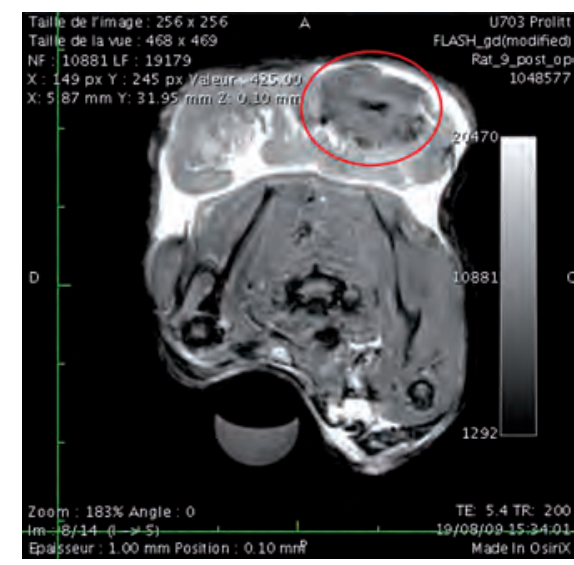

Figure 6. Nécrose de coagulation obtenue par un tir laser dans le modèle préclinique. Image obtenue par la microIRM du DHURE de Lille.

automatique des IRM multiparamétriques de la prostate. Ces travaux se poursuivent dans le cadre d'un projet financé par I'INCa déposé en réponse à I'AAP PAIR Prostate (2010-2012).

\section{Laser interstitiel thermique (LITT)}

Pour la modélisation et l'optimisation de cette technique, un modèle préclinique a été mis en place en collaboration avec les services du département hospitalo-universitaire de recherche expérimentale de Lille (DHURE) (figure 6). Les premiers travaux ont permis d'identifier les paramètres permettant d'avoir une reproductibilité dans le volume de nécrose obtenue [7].

L'expérimentation pré-clinique restant un moyen de test cher et lourd en termes de logistique, deux moyens de simulation ont été imaginés.

- La simulation in silico : les effets de l'interaction laser-tissu et la propagation de la chaleur ont été modélisés et simulés en utilisant des procédés mathématiques puissants (figure 7) [8]. En prenant en compte les propriétés du tissu prostatique, ces effets ont été simulés et corrélés aux résultats obtenus sur le modèle préclinique.

- La simulation sur fantôme : un fantôme anatomiquement valide, compatible avec l'imagerie IRM ainsi que l'imagerie échographique a été développé (figure 8). La prostate est fabriquée avec une composition spécifique lui permettant d'exhiber 
les mêmes propriétés optiques que le tissu prostatique [9].

Pour la mise en place clinique de cette technique, un partenariat est en préparation avec la société DEKA.

\section{Thérapie photodynamique interstitielle (iPDT)}

Depuis 2009, I'unité 703 collabore activement avec la société Steba BioTech dans le cadre des phases d'évaluation d'un nouveau photosensibilisateur. Cette collaboration concerne également le développement de nouvelles techniques de modélisation pour réaliser une planification dosimétrique qui garantit de déposer l'énergie nécessaire pour traiter la cible tout en épargnant les organes et tissus
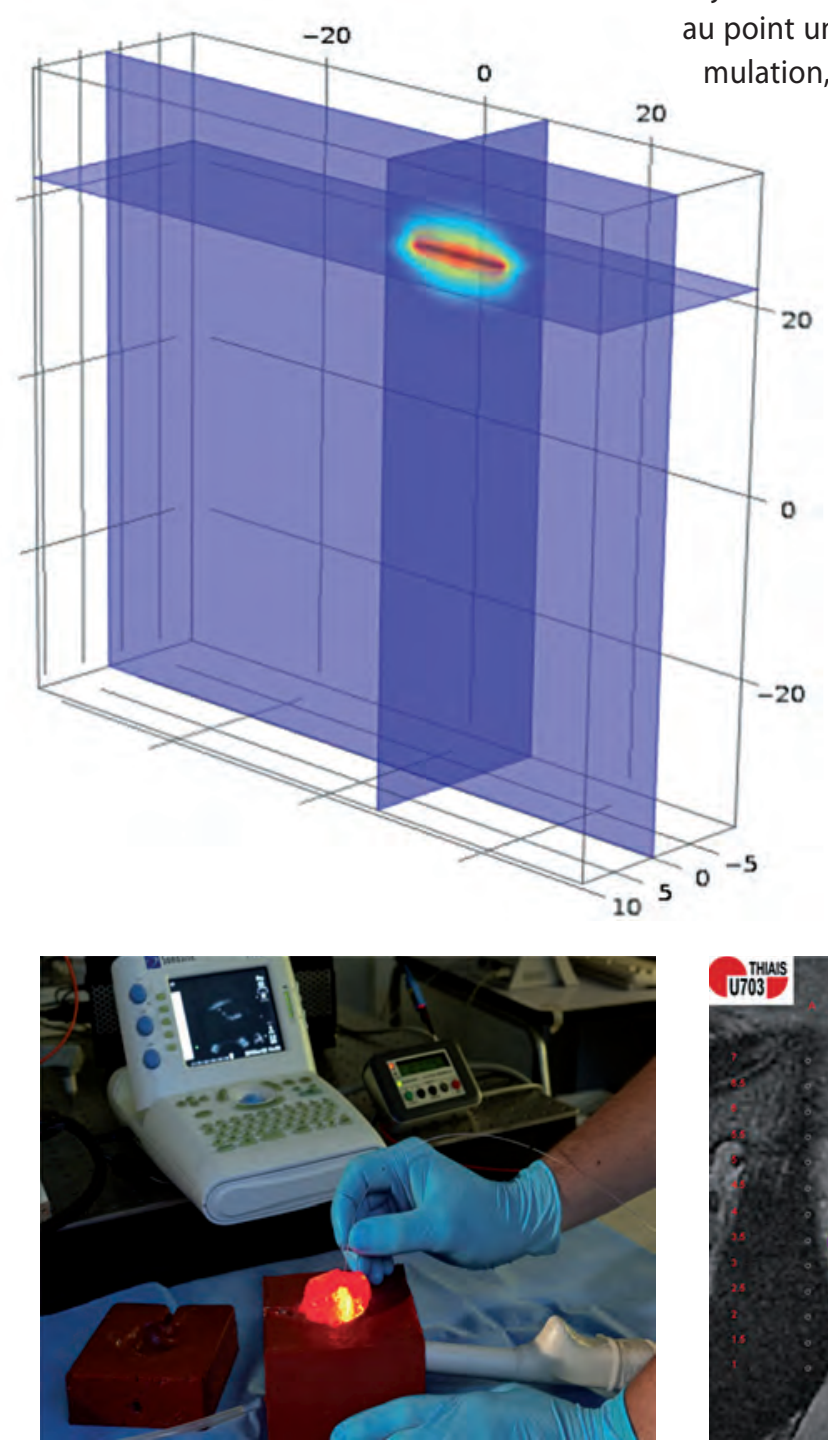

Figure 8. Simulation d'un tir laser guidé par imagerie échographique dans le fantôme (brevet InsermTransfert [9]).
Figure 7. Simulation des effets $d^{\prime}$ une fibre laser diffusante dans le tissu prostatique [8].

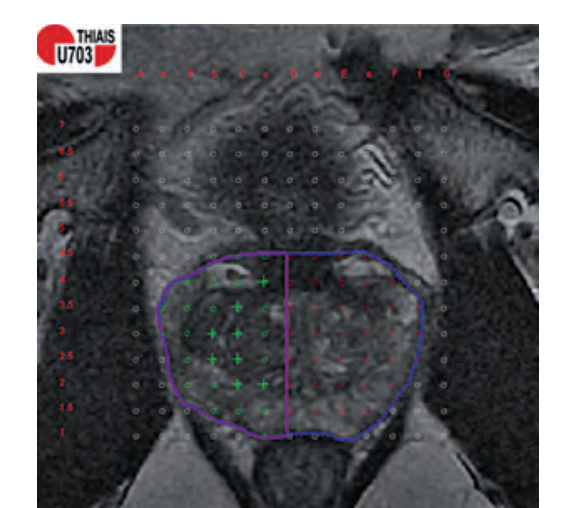

sains [10]. Ainsi et grâce à la mise à disposition des données issues des phases précédentes, une nouvelle méthode de planification a été mise au point et un logiciel a été développé (figure 9). Ce dernier fait partie intégrante du protocole d'évaluation de phase III multicentrique européen (2010-2014).

Aujourd'hui L'iPDT apparaît comme une technique alternative intéressante permettant de cibler de manière focale des lésions prostatiques. Cependant, elle nécessite encore des développements pour la rendre facilement applicable dans une routine clinique. La dosimétrie temps réel de l'iPDT permettant une planification efficace du traitement reste un challenge. L'objectif de l'étude en cours est de mettre au point une plateforme intégrée de siet de monitoring de l'iPDT. Les outils mis en place, permettront de moduler le traitement en fonction de la réponse mesurée en temps réel. Les expérimentations seront menées sur le modèle pré-clinique déjà utilisé. Ces travaux permettront de lever tous les verrous empêchant

Figure 9. Logiciel de planification dosimétrique pour la iPDT du cancer de la prostate [10]. cette technique de faire partie de l'arsenal thérapeutique du cancer. L'iPDT pourrait ainsi devenir une alternative accessible à tous les centres et non plus uniquement aux grands centres spécialisés.

\section{Références}

[1] Radical pleurectomy and intraoperative photodynamic therapy for malignant pleural mesothelioma. Friedberg JS, Ann Thorac Surg 2012; 93: 1658

[2] Development of a new illumination procedure for photodynamic therapy of the abdominal cavity. Guyon L, Lesage JC, Betrouni N, Mordon S, Journal of Biomedical Optics 2012; 17(3): 038001

[3] Innovative engineering design of a textile light diffuser for photodynamic therapy. Mordon S, Cohrane C, Lesage JC, Koncar V, Proceedings (N510) of the 13th IPA World Congress (May 10-14, 2011, Innsbruck, Austria) pp 147-151

[4] New design of textile light diffusers for photodynamic therapy. Cochrane C, Mordon S Lesage JC, Koncar V, Materials Science and Engineering $C 2013 ; 33(3): 1170$

[5] Photodynamic therapy of malignant brain tumours: A complementary approach to conventional therapies. Bechet D, Mordon SR, Guillemin F, Barberi-Heyob MA, Cancer Treat Rev 2012; doi: 10.1016/i.ctrv.2012.07.004

[6] Computer-assisted diagnosis of prostate cancer using DCE-MRI data: design, implementation and preliminary results. Puech $P$, Betrouni $N$, Makni N, Dewalle AS, Villers A, Lemaitre L, Int J Comput Assist Radiol Surg 2009; 4(1): 1

[7] Focal laser interstitial thermotherapy (LITT) at $980 \mathrm{~nm}$ for prostate cancer: treatment feasibility in Dunning R3327-AT2 rat prostate tumour. Colin P, Nevoux P, Marqa M, Auger F, Leroy X, Villers A, Puech P, Mordon S, Betrouni N, BJU Int 2012; 109(3): 452

[8] Focal laser ablation of prostate cancer: numerical simulation of temperature and damage distribution. Marqa MF, Colin P, Nevoux P, Mordon SR, Betrouni N. Biomed Eng Online 2011; doi: 10.1186/1475-925X-10-45

[9] An anatomically realistic and adaptable prostate phantom for laser thermotherapy treatment planning. Betrouni N, Nevoux P, Leroux B, Colin P, Puech P, Mordon S. Med Phy. 2013; 40(2): 022701

[10] A model to estimate the outcome of prostate cancer photodynamic therapy with TOOKAD Soluble WST11. Betrouni N, Lopes R, Puech P, Colin P, Mordon S, Phys Med Biol 2011; 56(15): 4771 